\title{
PRIM an Open Mobile Robot Platform. Motivation, Present and Future Trends
}

\author{
Lluís Pacheco, Joan Batlle, Xavier Cufí and Roger Arbusé \\ Computer Vision and Robotics Group \\ University of Girona. Technical School \\ Av. Lluís Santaló sn 17071 Girona, Spain \\ \{lluispa,xcuf\}@eia.udg.es
}

\begin{abstract}
This paper ${ }^{*}$ is focused on the robot mobile platform PRIM (Platform Robot Information Multimedia). This robot has been made in order to cover two main needs of our group, on one hand the need for a full open mobile robotic platform that is very useful in fulfilling the teaching and research activity of our school community, and on the other hand with the idea of introducing an ethical product which would be useful as mobile multimedia information point as a service tool.

This paper introduces exactly how the system is made up and explains just what the philosophy is behind this work. The navigation strategies and sensor fusion, where machine vision system is the most important one, are oriented towards goal achievement and are the key to the behaviour of the robot.
\end{abstract}

Index Terms - Open robot mobile system, Multimedia Mobile Information Point, Navigation Strategies Oriented to Achieve the Goal, machine vision system.

\section{INTRODUCTION}

Despite the enormous progress in robotics over the last half century, this field is very much in its infancy. Thus, robot behaviour is still simple when compared with human behaviour. Their ability to move, to understand complex sensorial inputs, or to perform higher level reasoning, is limited. The clues for achieve a renewed path of progress are the integration of several knowledgeable fields, such as computing, communications, and control sciences, in order to perform a higher level reasoning and use decision tools with strong theory base [1]. This work deals with some of these objectives through the creation of a low cost open mobile robot platform (PRIM-Platform Robot Information Multimedia). The philosophy of the robot includes two main goals which are the use of this platform as a full open educational tool, and their use as a mobile multimedia information point (MMIP), in commercial applications.

The educational goal should be considered under the roof of our university reality, where study and understanding in several academic fields such as electronics, mechanics, control, and computer science, can be reinforced by the use of these kinds of platforms. The use of such open platforms rather than other kinds of commercially available mobile robot, that act as closed platforms, where the control tasks of the users are constrained to programmed pre-

\footnotetext{
* This work was partially supported by the Grant-in-Aid of Scientific Research CICYT FOTOGEO (CTM-2004-04205) by the Spanish Ministry of Education, Science, and Culture, and by the Grant CERTAP -Virtual Prototypes in Construction Projects CERTAP-PVPC by the Catalan Government.
}

established commands is clear. Thus, students working with open platforms have the full platform access. Hence learning is attained in electronics, control and modelling, sensor fusion, and computer science among other areas. However this renewed path should not only be attained at first degree level, but it should also include research activities coming from the mutual interaction and integration between subjects, and the high level control strategies. The use of low cost open platforms allows the students to develop understanding in a multidisciplinary context

It is obvious that the design and implementation of such systems, is also of interest from a commercial point of view. Thus, this idea is not only restricted to the university community. The creation of an open ethic product is another of the objectives, and has been supported by Catalan association "Ateneu Informatic". In the context of this institution the idea of building a high performing low cost autonomous multimedia information tool began. The idea is fusing robotic technology, telecommunications, and computer science into just one technological product. The product must be ethical; such is the idea of having a double value: It is an MMIP, but it is also a declaration of principles, before the market incorporation. Their ethical and aesthetic characteristics should be defined, involving public administration, universities, and non governmental associations. In this context of an ethical product, the robot was presented at the 2004 Universal Culture Forum of Barcelona at the $18^{\text {th }}$ IAVE World Volunteer Conference from the $17^{\text {th }}$ to $21^{\text {st }}$ of August of 2004 , (see Fig. 1) [2]. To summarized, it was concluded that the ethical and aesthetic characteristics should cover all the needs that the third sector could possibly demand.

The particular motivation of this work arose from the following characteristics: platform flexibility, low cost, an open system, and multiple applications.

This work is organized as follows: In section I, the main ideas of this work, as an educational open mobile robot platform, and as an ethical and aesthetic MMIP have been presented.

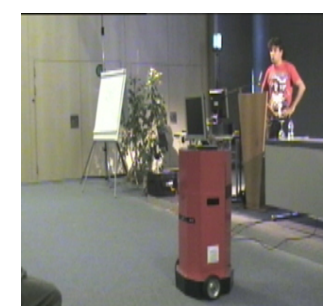

Figure1. The robot PRIM at the Universal Culture Forum of Barcelona 
Section II presents the main mechanics and electrical descriptions of the platform. The different parts are set up with the idea of flexibility, modularity, and low cost, as the main objectives of this open system. Within this context, the sensors, actuators, and the hardware architecture systems are briefly explained including the functionality description of each part. Hence, the hardware system sets up the capabilities of the robot.

Section III presents the philosophy of the control strategies, in the context of a flexible system, where the navigation strategies are set in order to achieve the goal. Within this context the different strategies that use different sensor systems related to the goal achievement are presented. The successful goal achievement could be improved in the context of the sensor fusion.

Section IV explains the machine vision system techniques used in this work that allow obstacle detection and incremental robot positioning relative to the acquired images.

Section V and VI presents the experimental results and the conclusions. The main problems found are also analysed, and the research into solutions that will give better results is considered over a wide range of topics that focused the future works on robotics.

\section{DESCRIPTION OF THE PLATFORM}

The robot PRIM is designed for indoor navigation, and previously gained knowledge from our experience in robotics was taken into consideration during its construction. follows:

The mechanical description could be summarized as

The actual structure of the robot is made from aluminium, as is shown in Fig. 2. It has four levels at where the different parts are placed. At the first level there are two differential drive wheels, controlled by two dc motors, and a third Omni-directional wheel that gives the third contact point with the floor.

The base of the robot has a size of $400 \times 550 \mathrm{~mm}$, and the hight is $800 \mathrm{~mm}$. The weigh of the robot is almost $20 \mathrm{~kg}$. On the low level the power stage is placed. On the second level there is the PC control computer. On the third level the specific hardware and the sonar sensors are placed. The forth level could be used, according to the flexibility of the system, to place the machine vision system or/and MMIP depending on the platform set up application.

The whole system can be powered by $12 \mathrm{~V}$ dc batteries or by an external power source through a $220 \mathrm{~V}$ ac.

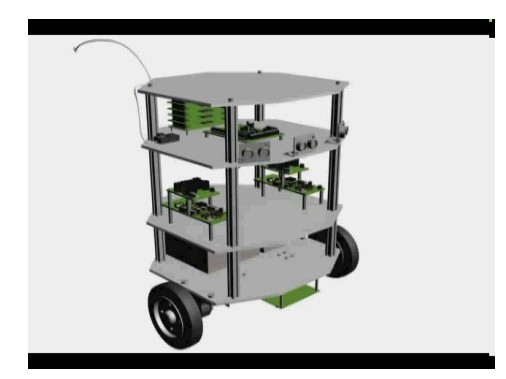

Figure 2. Basic hardware structure of the robot

The battery system is actually composed of a set of 4 batteries of $12 \mathrm{~V}$ with $7 \mathrm{Ah}$ each that provide between 3 and
4 hours of autonomy. The space remaining on the platform means that the system could be expected to have up to 8 hours of autonomy.

The robot is equipped with two de motors that act over the two independent traction wheels

The robot has the following sensorial system: Two encoders connected to the rotation axis of each dc motor, an array of sonar composed by 8 ultrasounds sensors, and the machine vision system.

The meaningful hardware consists of the following electronic boards:

1) The de motor power drivers based on a MOSFET bridge that controls the energy supplied to the actuators.

2) A set of PCB (printed circuits boards) based on PLD (programmable logic devices) act as interface between the PC system, the encoders, and the dc motors. The interface between the PLD boards and the PC is carried out by the parallel port.

3) A $\mu \mathrm{c}$ processor board controls the sonar sensors. The communication between this board and the PC is made through a serial port. This board is also in charge of a radio control module that enables the tele-operation of the robot.

4) The PC is the core of the basic control system, and it is where the high level decisions are taken.

The PLD boards generate the PWM (pulse width modulation) signals for each motor and the consequent timing protection during the command changes. This protection system provides a delay during the power connection, and at the change of the rotation motor sense. A hardware ramp is also implemented in order to give a better transition between command changes. The PLD boards also measure the pulses provided by the encoders, during an adjustable period of time, giving to the PC the speed of each wheel. The absolute position of each encoder is also measured by two absolute counters used in order to measure the position and orientation of the robot by the odometer system.

The system has two modes of operation tele-operated, and autonomous mode. In autonomous mode when the system starts running, the board disables the PLD boards until it has received from the serial port a control word. This prevents the noise reception going into the PLD boards, through the parallel port. Moreover, the $\mu \mathrm{c}$ has the control of the sonar sensors, so for each sensor a distance measure is obtained.

The flexibility of the system allows different hardware configurations as a function of the desired application and consequently the ability to run different programs on the $\mu \mathrm{c}$ or PLD boards. However, the platform is actually being tested under the configuration explained previously. The open platform philosophy is reinforced by the use of the similar $\mu \mathrm{c}$ and PLD boards that are used as teaching tools at our school. Fig. 3 shows the hardware architecture of the platform.

The main decision system arises from the PC that controls the hardware shown at this point. The software of the $\mathrm{PC}$ is implemented on $\mathrm{C}$ language and runs under the LINUX operative system. However, this platform acts as an open system that allows the connection of other PCs though a LAN. 


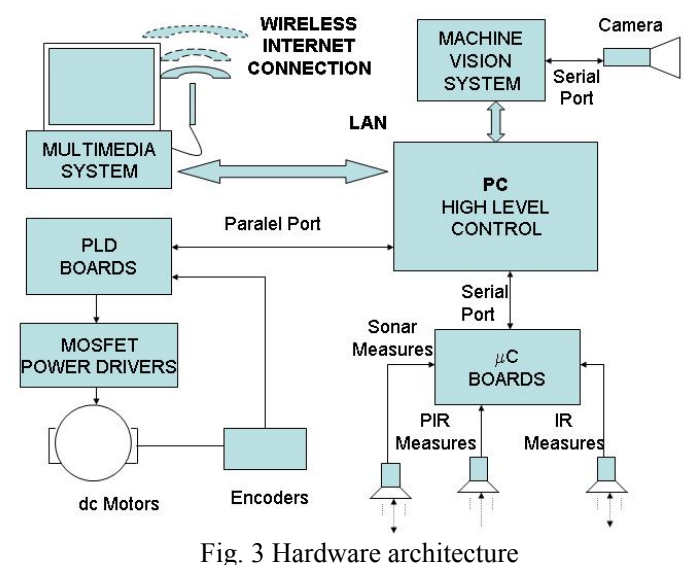

Fig. 3 Hardware architecture

These possibilities cover two complementary aspects of the system that of the multimedia point of information and the machine vision system, as an advanced sensor system.

The multimedia system is placed at the top level, and it is composed of a PC with a tactile screen that allows the interaction with people. The idea of this application as an MMIP means that this computer should be configured with the software information that the users applications demand. A wireless Internet connection allows the communication with the whole world, and a set of multiple possibilities.

The machine vision system goal is the sensor fusion of the high level image understanding with the information provided by other sensors. The machine vision system is composed of the following components: A remote camera with motorized focus, overture, and zoom control by a serial port, two steep motors that control the pan and tilt position of the camera, and specific hardware boards running on a $\mathrm{PC}$, exclusively used by the machine vision system. The system is connected to the main control system through a LAN.

\section{STRATEGIES OF NAVIGATION}

The NSOGA, (navigation strategies oriented to goal achievement) applied to PRIM are presented in this section. Thus, the goal achievement will set the sensors and strategies used.

The strategies tested and shown in this paper are mainly oriented to the navigation of the robot as an MMIP in a structured indoor environment. The work presented in this paper is based on navigation strategies with and without maps use.

The behaviour used for obstacle avoidance can be useful in order to avoid collisions in all the cases. Hence, the sensor fusion of the sonar and machine vision sensors is used.

\section{A. Navigation without maps}

When the navigation without maps is used, the system has no information about the scenario. Thus the movement of the robot is generated through the relevant information of the environment. The elements taken into account could be doors, walls, people, etc. The knowledge of the element positions is not necessary because navigation is implemented with respect to these elements. This kind of navigation, using sonar as sensors, was proposed in [3].

The robot can easily do various types of navigation using just the array of ultrasound sensors. These strategies include several behaviours such as tracking walls using with obstacle avoidance. The control strategies are set using fuzzy rules [4]. The fuzzy rules are based on the distance measured by each ultrasound. They provide a continuous actuation on the motors, with different norms, such as turn left, turn right, or go straight, that depending on the sensors measures are executed in a wide continuous range of speed values. Different behaviours such as right or left wall tracking, or equidistant navigation, have been studied. A more complete explanation of this work can be found [5].

The references [6], and [7], present the symbolic navigation based on using the command information as a source of information, and the necessary landmarks in order to find the path that should be followed in order to reach the final goal. These references deal with the NSOGA philosophy presented in this work, and it is considered by the authors as a very interesting, and important one.

The applications tested on the platform PRIM shows that it can perform some of these behaviours. Hence, navigation using sonar sensors has been presented.

The machine vision system can also be used as an important source of information in order to implement these kinds of behaviours. These techniques will be explained with more detail in the next section.

Navigation without maps, despite their simplicity, can be useful for the navigation as an MMIP in some scenarios. The detection and interaction with the people can be done by the means of obstacle detection. When this is done, the robot remains stationary waiting for an interactive action with the multimedia part. If no interaction with the robot is produced during a programmable period of time, the robot will then follow the navigation behaviour when the obstacle is not considered a person.

\section{B. Navigation with maps}

The type of navigation used in this case is incremental, so it is supposed that the initial position of the robot is known. Using these strategies the robot is able to move and execute the movement commands, and when the incertitude has a value bigger than a threshold, the sensors are able to be used in order to find a new fixed point. The localization of the robot comes from the topological description of the trajectory or from marks. A localization strategy based on the topological representation can be found in [8]. Thus, a graph is able to model with enough accuracy the information of the robot navigation map. The localization based on marks was proposed by several researchers. Therefore, in [9] a set of different patterns have been proposed in order to make the localization, and in [10] a set of line marks placed on the floor are used. The identification of natural marks should be one of the main objectives of our present and future research work [11]. Hence, it allows the navigation without the need of modelling the environment.

In this kind of incremental navigation with maps the odometer system seems to be the more adequate. Hence, the features of this system match the principles exposed before. However, the dead reckoning causes an incertitude in the odometer system, that can't be avoided, and thus, there is an accumulative error. The only way to solve this problem is the sensor fusion with other useful localization systems, as i.e. a machine vision system that can give enough 
information in order to find robust localization points in the navigation map.

Navigation using a simplified description of the map is very useful when working with NSOGA. Therefore, in this first stage of the work, the path is given as a set of description points on the map in the context of the configuration space [12]. This navigation is based on the description of the map as a set of points or nodes through which the robot should pass. From point to point the path is described by a straight line across the configuration space which is free of collisions. The collision risk with the obstacles is minimized by using the heuristic concept of the wide path motion [13]. Hence, the configuration path is obtained by increasing the obstacle size with the wide path motion in order to reduce the collision risk.

Giving the trajectories as a set of points, the control strategies consist in minimizing the orientation and position distance errors between the robot and the different segments of the trajectory. The control stability is guaranteed by the results of Hindman and Hauser. The distance probed was a Liapunov function and consequently there exists an asymptotic stability of the control system with respect to the desired trajectory [14]. The control strategies applied are heuristic that is for instance, the speed of the robot is set as a function of the distance to the change of orientation of the segments, and the differences of orientation and position between the path and the robot. The Fig. 4 presents the idea of the heuristics rules applied in order to reduce the orientation and position distances. The sensor system used in this kind of navigation is mainly the odometer system. However, once again, the incremental errors produced by the dead reckoning set the performance of this system. Thus, it is very useful when there are no great distances or not many orientation changes in the trajectory, but for long distances with many directional changes the incremental errors must be minimized by using extra sensors fusion information.

Once again, the use of machine vision systems in a sensor fusion context can give a lot of extra information. Hence, the orientation of the walls, the obstacle or landmark detection can help to improve the sensorial system.

\section{THE MACHINE VISION SySTEM}

Nowadays, the machine vision system is focused on monocular visual obstacle detection. We present an algorithm that has achieved good real world performance during many indoor tests.

The methodology presented in this issue pays special attention to image energy measure techniques using depth from focus (DFF) measures and optical flow (OFT) analysis. The motivation to use co-operative DFF and OFT methods lies in the fact that both allow a robust complementary set up. Hence, the OFT has many advantages when the image presents high brightness contrasts and there are straightforward edges.

The use of DFF techniques allows effective homogeneous floor detection even when there are shadows or brightness.

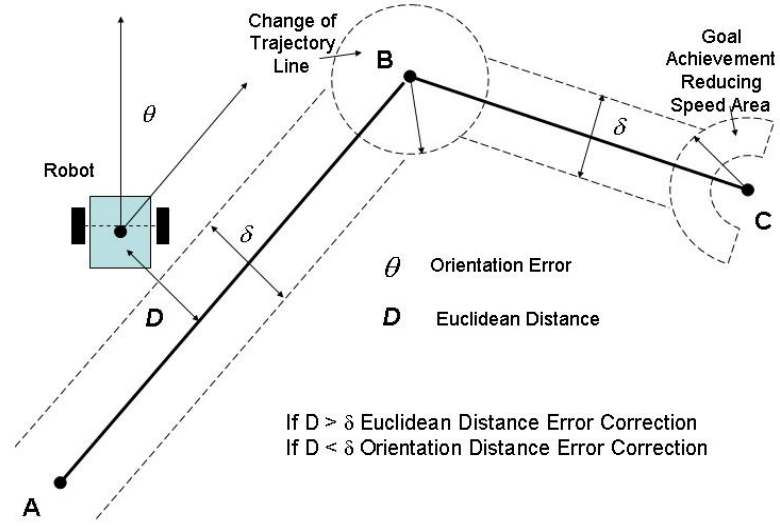

Fig. 4 Heuristic parameters in the simplified description of the path navigation

The use of pyramidal image resolution can speed up computations by reducing the search space. This technique is not only used as a way of reducing the information at its top level; thus it is possible to achieve a better environment understanding, using its bottom level information.

The machine vision system presented can be understood as a robust technique which, depending on needs and constraints, satisfactorily uses two different vision techniques. The interesting features of the system we present are simplicity, reliability, robustness, real time, and low cost.

The machine vision system algorithms implemented are based on two important assumptions that are generally accomplished in normal indoor environments and even in many outdoor scenarios. These constraints are flat surface navigation and homogenous floor energy.

The algorithm starts using the DFF techniques to measure the energy of the floor when no obstacle is placed in the field of view of the robot. When a valid energy floor measure is known, effective floor segmentation can be achieved. Thus, the image is analysed from bottom to top, searching for possible non-floor regions. Hence, feasible control unit information is provided with closer obstacle positions or free way detection. The OFT can discriminate obstacle validity when important floor energy discrepancies are met. A more accurate explanation of the algorithms used is showed in the following subsections.

A. DFF Algorithms. A Gaussian pyramid resolution is applied to the frames acquired in PAL format, $768 \times 576$ pixels. The pyramid algorithm description details can be found in [15]. This use allows noise reduction as well as a decreasing resolution that speeds up obstacle detection. Three decreasing resolution levels with different pixel sizes

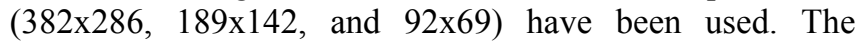
brightness image average is also computed. Thus, brightness normalization is carried out by dividing each image brightness pixel by the mean value [16]. Hence, better robustness is achieved when light variations are produced. The image energy is computed, over $3 \times 3$ windows at the top level of the pyramid, using the modified Laplacian method [17]. The standard deviation is another interesting statistic parameter that can also be used as it relates the homogeneity of the floor energy values. In most cases the process is computed only by using the top level information. Hence, the pyramid low pass filtering process improves floor 
homogeneity. The low level resolution is decreased using a $10 \times 7 \times 2$ array, where each cell represents the Laplacian mean value and the corresponding standard deviation, computed over 9x9 pixel patches on the $92 \times 69$ pyramid resolution level. The $10 \times 7 \times 2$ array is explored and the floor segmentation is carried out using both energy and standard deviation thresholds. It should be pointed that thresholds are a distance function. Then, if no obstacle is found, further processes are not used, and a new frame is acquired. Otherwise, if obstacles are encountered, optical flow techniques (OFT), explained in the next subsection, should be used.

B. OFT Algorithms The image brightness pattern motions observed by a camera, when it or some scene object is moving, is called optical flow. These techniques use a time varying sequence of images as a source of information. Unlike DFF methods, camera calibration is required, and accurate image point correspondence between acquired frames should be carried out. Reconstruction of 3D information through 2D information could also be considered as an ill posed problem [18]. Hence, it has been suggested, due to the inherent differences between 2D motion field and intensity variations, that usually only qualitative information can be extracted [19]. Despite this, the interesting features that optical flow presents have attracted the scientific community to develop a large number of quantitative methods.

The different quantitative approaches are generally based on two classical feasible assumptions, i.e. brightness constancy model (BCM) and optical flow smoothness. The $\mathrm{BCM}$ assumes that the brightness of the scene points, from sample to sample, remains constant or varies negligibly. Smoothness constraint is also assumed and this means that neighbouring object points have similar displacements. In this case, neighbouring image points will also have similar motions. Thus, image motion discontinuities are due to depth and motion discontinuity boundaries [20].

The OFT can solve two important objectives of robotics, i.e. motion detection and scene structure analysis. Thus, the robot's skill improvements can include interesting features, such as mosaic based positioning, visual servoing, structure from motion, or visual odometry, among others $[21,22,23,24]$. Obviously the applications and the assumptions, in terms of accuracy or constraints, will set the method performance.

Our proposal deals with the work of Campbell [24], but in our implementation, qualitative information knowledge is good enough to detect the obstacles. Furthermore, the use of the energy measures provided by DFF techniques makes the image feature matching and detection, and consequently the optical flow computation, easier. Thus, only the image obstacle regions detected using the energy measures should be computed. In this work, a Canny edge detector is proposed [25].

The use of wheel encoder-based odometer systems, in this stage of work, has made it unnecessary to implement an accurate visual odometer system. Thus, sensor fusion is proposed. Therefore, assuming that obstacles lie on the floor, the odometer system provides the robot positions from frame to frame, and DFF techniques provide obstacle detection on each frame. Thus, the corresponding obstacle floor contact coordinates can be easily matched from frame to frame and reduce the time of computation.

\section{EXPERIMENTAL RESULTS}

The camera used has a $43^{\circ}$ horizontal, and $36^{\circ}$ vertical, field of view. It is placed at the top of the robot looking down at the floor. The angle between the horizontal plane and the camera optical axis is $58^{\circ}$, and the height from the floor is $110 \mathrm{~cm}$. Using this configuration and the machine vision system obstacle avoidance techniques explained before, the system has not failed during the multiple indoor tests carried out since the summer of 2005. Fig. 5 shows obstacle detection based on the energy discrepancies between floor and obstacles. However, the sonar sensors have presented several obstacle detection failures, especially when there are obstacles out of sonar range (i.e. down stairs, small objects).

Sonar sensor information becomes interesting when the movement of the robot is out of the field of view of the camera. Thus, sonar measures give extra information that should be considered as a reactive control level useful as extra safety control or in some robot movements.

The odometer and machine vision system information fusion, related with OF obstacle matching, has been explained before.

Machine vision system obstacle detection is very useful when navigation strategies without maps are used. But their use with navigation maps is just at the beginning.

The knowledge of 3D obstacle positions, in contact with the floor, allows accurate path planning. The local knowledge provided by the camera field of view makes it impossible to find a global path to the goal. Thus, it can only be used as an obstacle detection system when fixed trajectories are used. Fig. 6 shows robot accuracy when a fixed trajectory should be repeated.

In addition, the detection of walls or other structured landmarks is just in the preliminary phase. Hence, wall detection experimental results, at this moment, are only restricted to textureless walls, and initial results are good. Thus, texture absence gives rise to less energy and standard deviation values. This allows walls to be easily detected.

Efforts are made to deal with control strategies that match the local information provided by the camera, and that allow the goal to be achieved in the context of NSOGA presented in this work. Thus, local model predictive control is reported [26].

The frequency of image computation is set between $5 \mathrm{~Hz}$, and $1.3 \mathrm{~Hz}$. This is good enough to control a mobile robot that moves at a maximum speed of $1.5 \mathrm{~m} / \mathrm{s}$, under the camera configuration presented.

As a conclusion, the machine vision system can be also very significant when working as a co-operative sensor with others.

\section{CONCLUSIONS AND FUTURE WORK}

The NSOGA has been presented and some strategies have been successfully implemented on PRIM. 


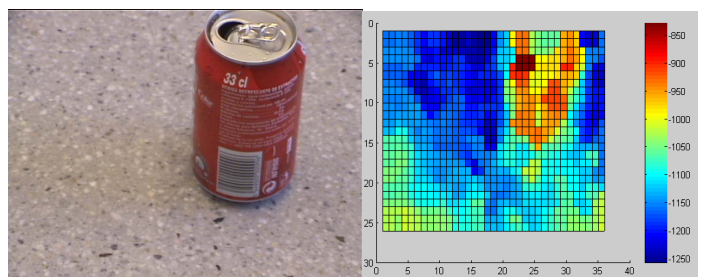

Figure 5. Obstacle detection using DFF techniques

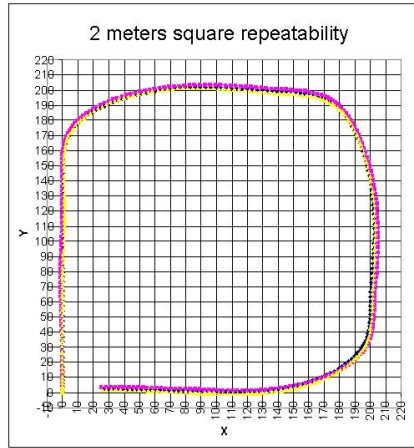

Figure 6. Experimentally results based on the odometer sensor system

The most interesting future work should be addressed to navigation using simplified maps. Localization using natural marks, such as doors or walls, in path navigation seems very interesting, and thus autonomous capabilities will be increased. This navigation strategy could be implemented by using different sensors. However, although the odometer system working in incremental navigation mode can be one of the most useful sensors, landmark localization becomes primordial. Thus, the incremental errors should be periodically set to zero and the main sensor used could be the machine vision system. Future work in the context of the machine vision system will be focused on path environment understanding.

It has been shown that by using OFT and DFF techniques obstacle detection can be done properly. But the real aim of this work is to implement NSOGA. Thus, the final objective is to use landmarks as the key to navigation on a simplified navigation map. Therefore, the platform must understand and perform high level commands, such as go straight for $20 \mathrm{~m}$, turn left, turn right at the third cross and then go through the second door on the left, etc. This allows the navigation in the context of symbolic object detection [6], and [7]. In such conditions an intelligent machine vision sensor would be absolutely necessary. Furthermore, the natural marks used by sonar sensor navigation can give extra information about robot orientation.

Future work should be aimed at improving the actual capabilities of PRIM, but the search for new solutions to the different problems in indoor environments should not be neglected. Only by searching for new applications and solutions, with the aim of improving robot capabilities by using sensorial integration, can the future applications of PRIM be guaranteed.

\section{ACKNOWLEDGMENT}

This work was supported by the Grant of Scientific Research CICYT FOTOGEO (CTM-2004-04205) by the Spanish Ministry of Education, and by the Grant CERTAPPVPC by the Catalan Government.

\section{REFERENCES}

[1] R. M. Murray, K. J. Aström, S. P. Boyd, R. W. Brockett, G. Stein, "Future Directions in Control in an Information-Rich World" IEEE Control Systems Magazine, pp. 20-33, April 2003.

[2] J. Izquierdo, J. Cifuentes, R. Arbusé, LL. Pacheco, "El Proyecto PRIM", 18th IAVE World Volunteer Conference Barcelona, page 73, Aug. 2004.

[3] H. R. Beom, H. S. Cho, "Sonar-based Navigation Experiments on a Mobile Robot in Indoor Environments" Proc. IEEE International Symposium on Intelligent Control, pp. 395-401, July 2000.

[4] L. A. Zadeh, "Fuzzy Sets, Information and Control" Vol. 8, pp. 338353, 1965.

[5] LL. Pacheco, X. Cufi, R. Garcia, "Navegación Autónoma del Robot Móvil Roger utilizando Control Fuzzy", Seminario de Electrónica Industrial, Automática, y Instrumentación, Matanzas (Cuba), Sept. 2001.

[6] D. Kim, R. Nevatia, "Symbolic Navigation with a Generic Map" Proc. IEEE Workshop Vision for Robots, pp. 136-145 Aug. 1998.

[7] D. Kim, R. Nevatia, "Recognition and Localization of Generic Objects for Indoor Navigation Using Functionality" Image and Vision Computer, Vol. 16, no. 11, pp. 729-743, Aug. 1998.

[8] B. J. Kuipers and Y. T. Byun, A Robot Exploration and Mapping Strategy Based on a Semantic Hierachy of Spatial Representation, J. Robotics and Autonomous Systems, vol. 8, pp. 47-63, 1991.

[9] M. R. Kakuba; A. E. Arenas, Position Verification of a Mobile Robot Using Standart Pattern, IEEE J. Robotics and Automation, vol. 3, no. 6, pp. 505-516, Dec. 1987.

[10]T. Tsumura, "Survey of Automated Guided Vehicles in Japanese Factory" Proc. Int'l Conf. Robotics and Automation, pp. 1329- 1334, Apr. 1986.

[11] M. Hashima, F. Hasegawa, S. Kanda, T. Maruyama, T. Uchiyama, "Localization and Obstacle Detection for a Robot Carrying Food Trays” Proc. IEEE Int'1 Conf. Intelligent Robots and Systems, pp. 345351, Sept. 1997.

[12] T. Lozano-Perez, "Spatial planning: A configuration space approach," IEEE Trans. Computers, Vol. C-32, pp. 108-120, 1983.

[13]R. J. Schilling, Fundamental of Robotics, Prentice-Hall International, 1990.

[14]R. Hindman and J. Hauser, "Maneuver modified trajectory tracking." In: Proceedings of MTNS'96, International Symposium on the Mathematical Theory of Networks and Systems, St Louis, MO, USA 1992.

[15]E. H. Adelson, C. H. Anderson, J. R. Bergen, P. J. Burt, J. M, Ogden, "Pyramid Methods in Image Processing", RCA Engineer, 29-6, pp. 3341, Nov/Dec. 1984

[16]G. Surya,, "Three Dimensional Scene Recovery from Image Defocus", PHD thesis, Stony Brook, New York, december 1994.

[17] S. K. Nayar, Y. Nakagawa, "Shape from Focus", IEEE transactions on PAMI, Vol 16. No. 8, August 1994.

[18] Y. Aloimonos, Z. Duric, "Active Egomotion Estimation: a Qualitative Approach", Proc. ECCV, Ligure, Italy, pp. 497-510, 1992.

[19]A. Verri, T. Poggio, “Against Quantitative Optical Flow”, Proc. IEEE ICCV, London, pp. 171-180, 1987.

[20]S. Negahdaripour, Video Analysis and Optical Flow Computation, Lecture Notes on, October 2004.

[21]R. Unnikrishnan, A. Kelly, " Mosaicing large Cyclic Environments for Visual Navigation in Autonomous Vehicles”, IEEE ICRA, Vol. 4, pp. 4299-4306, May 2002.

[22] R. Vidal, O. Shakernia, S. Sastry, "Formation Control of Nonholomic Mobile Robots with Omnidirectional Visual Servoing and Motion Segmentation", IEEE, ICRA, pp. 584-589, 2003.

[23]F. Dellaert, S. M. Seitz, C. E. Thorpe, S. Thrun, "Structure from Motion without Correspondence", IEEE Conf. on CVPR, June 2000.

[24] J. Campbell, R. Sukthankar, I. Nourbakhsh, A. Pahwa, "A Robust Visual Odometry and Precipice Detection System Using Consumergrade Monocular Vision", Proc. of the IEEE ICRA, Barcelona, April 2005.

[25] J. Canny, “A Computational Approach to Edge Detection", IEEE Trans. On Pattern Analysis and Machine Int., Vol. 8, No. 6, Nov. 1986.

[26] LL. Pacheco, N. Luo, R. Arbuser, "Modeling and Control Strategies on an Open Mobile Robot Platform PRIM", “unpublished”, AQTR 2006. 